\section{Spreading Phenomenon and Inflammation}

A study has been made of the spreading activity of certain inflammatory agents in rabbit skin. As the results of this work may be of general interest, a brief account of them are given here.

Using hæmoglobin as an indicator of spreading, it has been found that the intradermal injection of inflammatory agents into the shaved skin of anæsthetized rabbits produces significant spreading as compared to saline controls. The agents investigated (concentration in $\mathrm{mgm}$. per c.c.) were the following : trypsin (10), chymotrypsin (10), 'Bactopeptone' (100), Wittes' peptone (100), casein digest (100), $\frac{1}{2}-\frac{1}{4}$ saturated urea, and snake venoms $(0 \cdot 1)$ (copperhead, diamondback rattlesnake and mocassin), wherein the hyaluronidase originally present was destroyed by treatment with alkali ${ }^{1}$.

The spreading observed with these inflammatory agents differs from that produced by hyaluronidases in that the rate of spreading is slow and the injected bleb persists as spreading proceeds. When these agents are injected into the skin of recently killed rabbits, where inflammatory œdema cannot occur, no spreading response is observed. This demonstrates that inflammatory agents do not directly alter the permeability of the dermal barrier to fluid diffusion. To determine whether the inflammatory agents acted via liberation of 'bound' hyaluronidase in skin', the effect of a hyaluronidase inhibitor upon the spreading activity of inflammatory agents in live rabbits was evaluated. Nitrated and partially de-esterified nitrated hyaluronates which markedly reduce hyaluronidase viscosity-reducing activity by competitive inhibition $^{3}$ were used in these experiments. It was observed that whereas the spreading activity of purified testis hyaluronidase or of snake venoms containing hyaluronidase was either inhibited or abolished by the inhibitors, the spreading activity of these inflammatory agents uniformly was not affected.

The mechanism by which inflammatory agents produce spreading in skin is explicable in terms of simple mechanical forces. It has previously been found that the area of spread produced by saline hæmoglobin mixtures is proportionate to the volume of fluid injected, or on the interstitial fluid pressure of the $\mathrm{bleb}^{2}$. Since inflammatory agents produce cedema consequent to capillary damage in the injected area, this increases the interstitial pressure of the bleb, thus giving rise to greater spreading than is observed with the saline control. This view explains why the injection bleb persists (or even becomes higher), in association with spreading of the indicator, with these agents in contrast to the spreading seen with hyaluronidase or saline, where the bleb flattens as spreading proceeds. It should be emphasized that the permeability of the dermal barrier need not be affected in these acute inflammatory spreading reactions.

These findings may provide an explanation for the fact that diverse agents ranging from simple chemicals like arsenious oxide to biological factors present in some bacteria and venoms, while without influence upon hyaluronic acid in vitro, nevertheless possess significant spreading activity in vivo ${ }^{4}$. The suggestion is advanced that these spreading factors, some of which are known to be inflammatory agents, act via the capillary damage they induce. In this connexion, it may be important to note that the spreading activity of extracts of rabbit organs such as spleen, lung, and liver (prepared according to the method of Claude and Duran-Reynals ${ }^{5}$ ), is similar to the inflammatory agents studied, in that the slow spread obtained with these tissue extracts is not inhibited by nitrated hyaluronates, and the spreading response is absent, in the skin of dead rabbits.

In previous studies it was demonstrated that inflammatory agents present in snake venoms markedly increase the final area of spread produced by purified testis hyaluronidase ${ }^{1}$. It has now been observed that this augmentation of spread can be duplicated by non-specific inflammatory agents such as concentrated urea and peptones. In experiments where a constant hyaluronidase concentration was injected with variable concentrations of inflammatory agents (peptones, proteolytic enzymes and alkali-treated snake venoms), it has been observed that the final area of spread is proportional to the concentration of inflammatory agent employed.

These studies illustrate the importance of inflammation in spreading phenomenon and may have implications for local bacterial invasiveness. It would appear that bacteria which do not produce hyaluronidase may spread extensively, although less dramatically than hyaluronidase-producing organisms, via the inflammatory changes they induce. The invasiveness of hyaluronidase-producing bacteria may depend quantitatively upon the degree and character of the inflammation they induce, and only secondarily upon the concentration of hyaluronidase produced in situ.

Full details of these studies will be published elsewhere. This work was aided in part by a grant from the G. D. Searle Company. \author{
SIDNEY SolomoN \\ Worcester Foundation for \\ Experimental Biology, \\ Shrewsbury, Mass., \\ and \\ Department of Physiology, \\ Tufts Medical School, \\ Boston, Mass. \\ May 8. \\ ${ }^{1}$ Hechter, O., J. Exp. Med., 85, 77 (1947). \\ 'Meyer, K. Chaffee, E., Hobby, G. L., and Dawson, M. H., J. Exp. \\ Med., 78, 309 (1941). \\ ${ }^{3}$ Hadidian, Z., and Pirie, N. W., Biochem. J., 42, 266 (1948). \\ ¿ Duran-Reynals, F., Bact. Rev., 6, 197 (1942). \\ ${ }^{5}$ Claude, A., and Duran-Reynals, F., J. Exper. Med., 60, 457 (1934).
}

Oscar Hechter

\section{Destruction of Urinary Glucuronide by Bacteria}

IN a recent publication, Senior ${ }^{1}$ has emphasized the importance of the preservation of rabbit urine preceding estimations of its glucuronide content. This point has been previously made by, for example, Venning ${ }^{2}$ and by Bucher and Geschickter ${ }^{3}$ with regard to pregnanediol glucuronide in human urine, and has lately been further investigated ${ }^{4}$.

We have carried out experiments to try to de. termine what organisms may be responsible for the apparently complete destruction of 'pregnanediollike-glucuronide ${ }^{/ 4}$ which may occur in unpreserved human urine. We have found that, whereas a number of common bacteria were without effect on this substance, its destruction, as well as that of added sodium pregnanediol glucuronidate, occurred when certain strains of Staph. albus were grown in 